
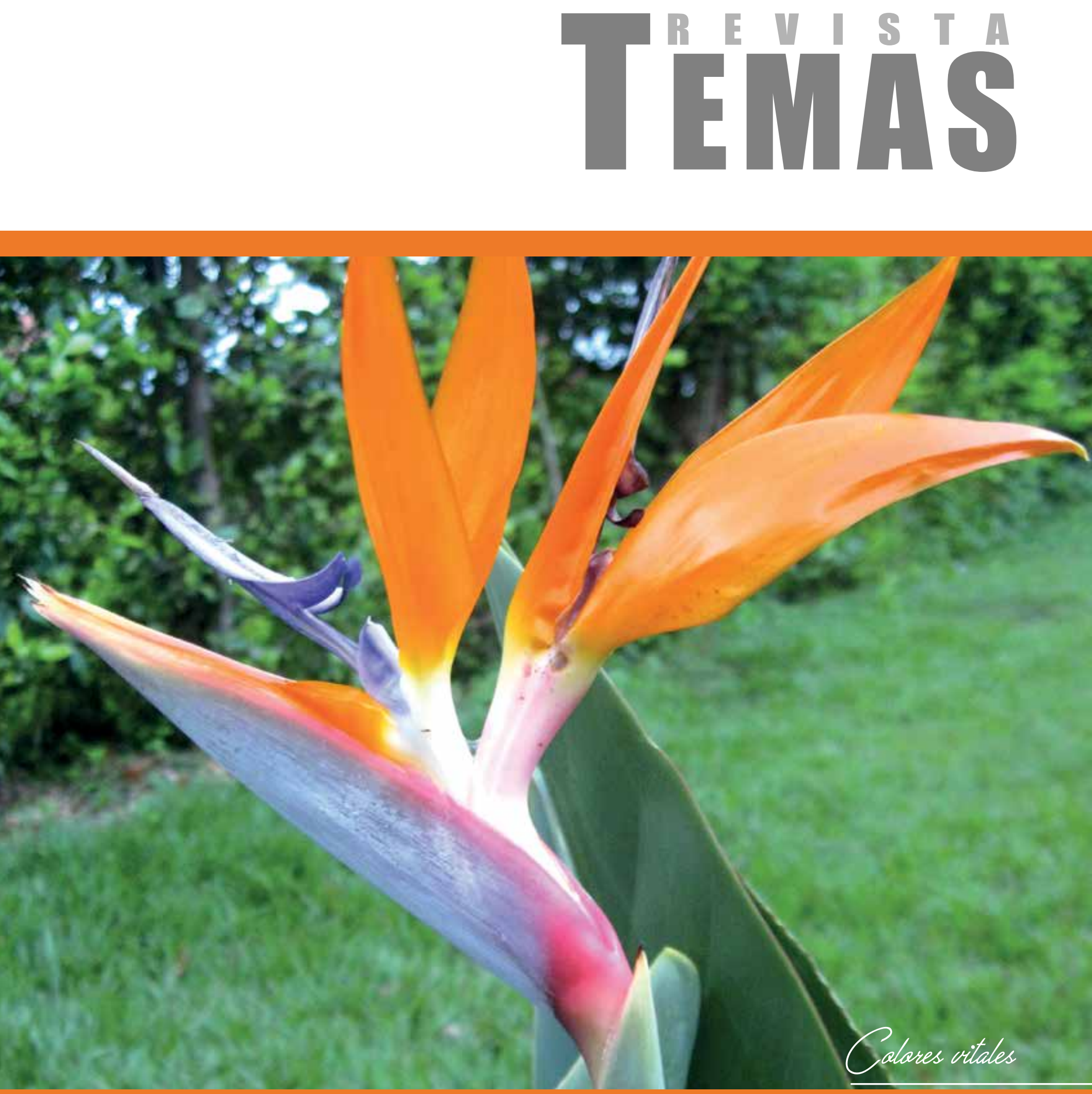

Referencia al citar este artículo:

Cárdenas, A. (2016). Fomentando la paz a través del fútbol y otros deportes en contextos de conflicto: el caso de Colombia e Irlanda del Norte. REVISTA TEMAS, 3(10), 51 - 62.

\title{
Fomentando la paz a través del fútbol y otros deportes en contextos de conflicto: el caso de Colombia e Irlanda del Norte ${ }^{1}$
}

\author{
Alexander Cárdenas ${ }^{2}$
}

Recibido: 2/19/2016

Aceptado: 3/11/2016

\section{Resumen}

Este artículo explora el uso del deporte para la construcción de paz en Colombia e Irlanda del Norte, desde la perspectiva del personal involucrado en el diseño e implementación de programas de deporte y paz. Haciendo especial hincapié en las reflexiones y puntos de vista de este personal, el contenido de programas sociales basados en el deporte y el sector del deporte para la paz y el desarrollo en las dos sociedades, este estudio extrajo una serie de lecciones referentes a las oportunidades y los retos que presenta la práctica del deporte para la promoción de la paz dentro del contexto específico del conflicto en las dos regiones.

\section{Palabras clave}

Deporte para el desarrollo y la paz, deporte y construcción de paz, Colombia, Irlanda del Norte.

\section{Fostering peace through football and other sports in Colombia and Northern Ireland}

\section{Abstract}

This article explores the use of sport for peace-building in Colombia and Northern Ireland from the perspective of the personnel involved in the design and implementation of sport for peace programs. With particular emphasis on the reflections of sport for peace officials, the content of sport-based social programs and the sport for development and peace sector in the two societies, this study drew a number of lessons regarding the opportunities and the challenges of using sport to promote peace in the specific conflict context of the two regions.

\section{Keywords}

Sport for development and peace, sport and peace building, Colombia, North Ireland.

\footnotetext{
1 Este artículo de investigación proviene de la tesis doctoral realizada por el autor.

2 Es consultor internacional, docente e investigador en el área del deporte para la paz y el desarrollo. Recibió un doctorado en estudios de paz, conflicto y desarrollo en la Universidad Jaume I de Castellón, España. Correo electrónico: alexcarmen@yahoo.com
} 


\section{Introducción}

En años recientes, el deporte ha obtenido un reconocimiento sin precedentes como un vehículo legítimo para promover la cohesión social, en especial, en sociedades que experimentan pobreza, violencia y conflicto. Partiendo del principio de que el deporte posee ciertas características que facilitan el logro de objetivos sociales amplios, incluyendo la construcción de la paz en escenarios altamente volátiles (Cárdenas, 2013; Schulenkorf, 2010), una multiplicidad de actores sociales, tales como los gobiernos nacionales, las organizaciones no gubernamentales (ONG), las agencias de cooperación, las instituciones académicas, las federaciones deportivas y el sector privado, han incorporado el deporte como un componente importante de sus agendas (Grupo Internacional de Trabajo para el Deporte, el Desarrollo y la Paz SDP IWG, 2008). Este enfoque se conoce como el Deporte para el Desarrollo y la Paz (DDP) y ha surgido no solo como una estrategia popular de intervención social, sino también como un sector específico dentro de la política pública (Giulianotti, 2011b), como un movimiento social (Kidd, 2008) y como un área académica interdisciplinaria en expansión.

El reconocimiento al deporte como una estrategia de intervención social se refleja en un aumento en el número de organizaciones, programas e intervenciones que utilizan el deporte como componente central de sus actividades. Donelli estima que para el 2007 aproximadamente 400 ONG operaban en el campo del DDP, con un promedio de diez más creadas mensualmente (citado en Giulianotti y Armstrong, 2011, p. 379). Del mismo modo Lyras et al., 2009 registran un incremento en el número de proveedores y proyectos de deporte para el desarrollo: de 200 en el 2005 a más de 1500 en el 2009 (citado en Kay y Dudfield, 2013); solamente en el
2012 se publicaron 25 artículos, capítulos de libro y tesis (Hillyer, 2013).

A pesar de posicionarse como un campo académico interdisciplinario en franca expansión, en la literatura actual sobre el papel del deporte y su vínculo con la paz en contextos de conflicto escasean las investigaciones que: 1) exploren las experiencias y reflexiones del personal a cargo del diseño e implementación de proyectos de deporte y paz; 2) incorporen estudios de caso inter-regionales que se extiendan más allá de África, el Medio Oriente y Europa del Este; 3) examinen el contenido de programas del DDP y 4) provean una visión general del sector del DDP de las sociedades donde estos programas son implementados.

\section{Propósito del estudio}

Con el objetivo de abordar los anteriores cuatro argumentos, el presente estudio pretende contribuir al campo académico interdisciplinario del DDP al explorar el uso del deporte recreativo (en especial del fútbol en su condición de deporte más popular del planeta) como un vehículo para fomentar la paz en sociedades en conflicto -a saber, Colombia e Irlanda del Norte-. Esto, desde la perspectiva de la investigación para la paz y visto a través de la óptica del personal involucrado en el diseño, la implementación y el soporte de programas de Deporte para el Desarrollo y la Paz (funcionarios del DDP).

\section{Contexto}

Tanto Colombia como Irlanda del Norte han experimentado recientemente un aumento en la actividad del DDP y así para promover la paz y, potencialmente, para abordar problemáticas específicas relacionadas con la dinámica de sus conflictos particulares. A través de una serie de programas e intervenciones sociales basadas en el deporte, una amplia variedad de actores locales e internacionales 
usan el deporte recreativo para convocar a jóvenes y niños (y a la comunidad en general) para promover la resolución pacífica de las diferencias, contribuir a la promoción de la paz y aportar a los procesos de desarrollo personal y comunitario.

En el caso de Irlanda del Norte, una serie de organizaciones emplean el deporte como una estrategia válida para fomentar la reconciliación entre católicos y protestantes, permitiendo, a través de esta actividad, la formación de relaciones significativas entre miembros de estas comunidades. Mientras que el discurso popular percibe la lucha de Irlanda del Norte exclusivamente como un enfrentamiento entre católicos y protestantes, se trata en realidad de un conflicto de reclamaciones e intereses por parte de aquellos cuyas lealtades están con la República de Irlanda, y aquellos que son leales al Reino Unido (McGlynn, 2010, p. 69). A pesar de que un acuerdo de paz fue firmado hace más de quince años, esta confrontación étnica y política ha dejado divisiones profundas que todavía son visibles hoy en áreas tales como la recreación y el deporte. Aunque es evidente que hay muchas excepciones a esta regla, a grandes rasgos, el deporte en Irlanda del Norte se expresa generalmente en términos de identidad cultural; es decir, el tipo de deporte practicado y los equipos apoyados, generalmente revelan la identidad religiosa (y posiblemente la afiliación política) de un individuo (McGinley et al., 1998, pp. 464-471), lo que puede contribuir a la expansión de la brecha divisoria entre ambas comunidades.

En Colombia -donde un proceso de paz actualmente busca poner fin al conflicto armando más antiguo del hemisferio occidental- los esfuerzos para promover la paz no se limitan a la búsqueda de una solución política de las hostilidades. Un movimiento por la paz -en gran medida asociado con la sociedad civil- busca la movilización de todos los sectores de la sociedad colombiana para actuar a favor de la paz a través de una variedad de esfuerzos e iniciativas. Cada vez más, las expresiones culturales y artísticas y sobre todo el deporte, han sido reconocidos por los líderes políticos, las organizaciones internacionales y la sociedad civil como aliados poderosos para avanzar en la construcción de la paz en la nación ${ }^{3}$.

El interés en explorar el papel del deporte como una herramienta para promover la cohesión social dentro del contexto particular del conflicto en Colombia está ganando impulso. Prueba de ello es el aumento en el número de programas e intervenciones que utilizan el deporte como un componente principal de sus actividades, encaminadas, en gran medida, a promover la paz en comunidades afectadas por la violencia y el conflicto. También se ha registrado un evidente aumento en los reportes de prensa, programas radiales y de televisión que informan al público sobre este fenómeno emergente. De especial interés ha sido el uso del deporte recreativo en programas sociales con el objetivo de promover la cohesión social y contribuir a los esfuerzos encaminados a la construcción de la paz en la nación (Cárdenas, 2012b; 2013).

\section{Metodología}

Este estudio adoptó un enfoque cualitativo de investigación (Creswell, 2003), empleó estudio de caso colectivo como metodología (Stake, 2000) y la triangulación como método de análisis de datos (Yin, 2004).

\section{Participantes}

En la selección de los participantes para este estudio se tuvo en cuenta el siguiente criterio: a) Funcionarios involucrados en el diseño, apoyo y ejecución de actividades de deporte y paz en Irlanda

3 Ver: Sport for peace in a post-conflict Colombia (2015); Sport, the international community and the peace talks in Colombia (2015). 
del Norte y Colombia, que representan las organizaciones y programas de mayor visibilidad en la promoción de la paz a través del deporte en ambos países: (ONG nacionales e internacionales, agencias gubernamentales, agencias de cooperación internacional, federaciones deportivas, instituciones del sector privado e instituciones académicas) y b) organizaciones o programas que emplean el fútbol recreativo -como deporte principal o como uno de los deportes de apoyo-para lograr sus objetivos sociales, especialmente aquellos relacionados con la construcción de paz.

En Colombia treinta funcionarios de veinte organizaciones o programas del DDP participaron en este estudio: A Ganar (programa bandera de la ONG internacional Partners of the Americas), Agencia Alemana de Cooperación, Banco Mundial, Con-Texto Urbano (asociación ONG), Colombianitos (ONG), Federación Colombiana de Fútbol, Fundación Luker, Fórmula Sonrisas (ONG), Fútbol Con Corazón (ONG), Gestores del Deporte (programa de deporte y convivencia de Coldeportes - Departamento Administrativo del Deporte la Recreación y la Actividad Física), Goles por la Paz (ONG), Golombiao (programa estrategia del Sistema Nacional de la Juventud), Grupo Internacional de Paz (ONG), Tiempo de Juego (ONG), Universidad Militar, Programa de las Naciones Unidas para el Desarrollo - PNUD, Peace and Sport y Children International (ONG internacionales), Fondo de las Naciones Unidas para la Infancia - Unicef, World Coach Colombia (ONG).

En Irlanda del Norte 16 oficiales de 9 organizaciones y programas del DDP contribuyeron en esta investigación: Asociación Norirlandesa de Fútbol, Derry City Council (Alcaldía de Derry), Derry/ Londonderry YMCA (ONG internacional), Football for Peace (ONG internacional), Northern Ireland Street League (programa comunitario), Peace Players International
(ONG internacional), Seaview Enterprises (programa del sector privado), Teenage Kickz (ONG), Universidad de Ulster.

\section{Procedimiento}

Los datos fueron recogidos entre marzo de 2012 y junio de 2014 en Colombia e Irlanda del Norte, a través de entrevistas abiertas, encuestas en línea, observaciones directas, observación participante y análisis de documentos. El análisis de los datos recogidos en este estudio empleó dos métodos: Análisis de caso y Análisis cruzado de casos (Merriam, 1998; Yin, 2009). Siguiendo el protocolo establecido por Creswell (1998), se encontró un conjunto preliminar de categorías, el cual se basó en patrones repetidos a lo largo de la encuesta en línea, las entrevistas y las observaciones. La interpretación de los datos recolectados se hizo basado en tres dimensiones: Funcionarios del DDP, programas del DDP y el sector del DDP. En esta etapa, ambos casos se exploraron a fondo, identificando correspondencias y divergencias en el uso del deporte para la paz en Colombia e Irlanda del Norte.

\section{Hallazgos y discusión}

\section{Dimensión I: Funcionarios de programas del DDP}

Esta categoría exploró: 1) Las opiniones de los funcionarios DDP en cuanto a las ventajas y limitaciones que presenta el deporte para alcanzar los objetivos de sus organizaciones; 2) el papel del deporte dentro del contexto del conflicto y, 3) sus reflexiones como "constructores de paz" y como promotores de cambio comunitario.

1) Los funcionarios del DDP en Colombia e Irlanda del Norte reconocieron la importante función que el deporte cumple para alcanzar los objetivos de paz de sus organizaciones. A juicio de estos funcionarios, el deporte: 1) 
Sirve como herramienta educativa $y$, 2) como plataforma para transmitir valores positivos aplicables más allá de los campos de entrenamiento; 3 ) actúa como un medio efectivo y fiable para atraer participantes a sus programas, y 4) logra traspasar las barreras sociales y culturales. Además, los valores relacionados con el deporte y sus lecciones constituyen una herramienta constructiva para ayudar a los niños y jóvenes a mejorar sus vidas y a transformar sus comunidades.

La idea del deporte como medio educativo fue más popular entre los oficiales DDP en Colombia:

El fútbol tiene una gran capacidad para facilitar el logro de muchas cosas... Encontramos enfoques explícitos que enseñan a la gente a resolver los conflictos, tales como la Metodología Fútbol por la Paz (especialista en educación, Banco Mundial).

A diferencia de sus homólogos colombianos, los funcionarios DDP norirlandeses se mostraron más inclinados a resaltar al deporte como una herramienta para involucrar a participantes en sus programas de paz y cohesión social:

El deporte es para nosotros un gancho. Al final no importa realmente qué deporte vamos a utilizar. En algunos casos el baloncesto tiene sentido porque es neutral. En otro casos, sea cual sea el deporte más atractivo que consiga reunir a los niños, es este el que se debe utilizar (Director, Peace Players International).

Los funcionarios del DDP apoyan la idea de que el deporte ofrece varias ventajas como estrategia de intervención social. Sin embargo, la mayoría de los funcionarios entrevistados señalaron varias limitaciones e inconvenientes relacionados con esta práctica. Algunos de los oficiales transmitieron la idea de que sería erróneo suponer que todos los niños y jóvenes están intrínsecamente interesados en el deporte. En Colombia, por ejemplo, se enunció repetidamente que la falta de espacios adecuados para la práctica deportiva y la poca conciencia sobre la dimensión social del deporte, podría tener efectos negativos a la hora de promocionar e implementar intervenciones sociales basadas en el deporte.

2) La información recogida indica que los funcionarios DDP apoyan la idea de que el deporte puede ser útil para abordar parcialmente algunos de los desafíos que son consecuencia del conflicto de cada país. En Irlanda del Norte, el deporte recreativo es visto como un medio para abordar dos objetivos principales, en orden de importancia: 1) Promover el contacto entre católicos y protestantes y 2) fomentar un mayor entendimiento intercultural entre niños y jóvenes de ambas comunidades. El primer objetivo se vincula con la idea de que la interacción entre individuos de grupos en oposición -cuando se reúnen ciertas condiciones- puede conducir a una disminución significativa del perjuicio y las hostilidades (Allport, 1954). Este ha sido uno de los principios rectores de diversas iniciativas del DDP en Irlanda del Norte, incluida la ONG Teenage Kickz:

A través del deporte hemos construido puentes entre ambas comunidades, ya que en el pasado había una especie de animosidad constante entre jóvenes protestantes y católicos (Director, ONG Teenage Kickz).

Del mismo modo, los funcionarios DDP manifestaron que los programas deportivos podrían tener un efecto positivo en el aumento de la sensibilización intercultural entre jóvenes y niños en ambos lados de la brecha sectaria. Dado que el sistema escolar y el esquema de vivienda son altamente segregados (Cairns \& Hewstone, 2002), pupilos católicos y protestantes viven, crecen y van a 
los colegios casi exclusivamente con los miembros de su propia comunidad. En este contexto, el deporte se utiliza para “... construir una comprensión de su propia cultura y construir una comprensión de la cultura de otras personas", aseguró un funcionario de la Universidad de Ulster.

La presente investigación también corroboró la condición política del deporte en Irlanda del Norte. Por medio de observaciones $y$, específicamente, a través de testimonios de los oficiales del DDP, se ha confirmado que el deporte en esta sociedad desempeña un doble papel: Puede ser un elemento unificador y un promotor de la conciencia intercultural, pero si no se gestiona adecuadamente, podría exacerbar las animosidades entre las dos comunidades. En Irlanda del Norte, según lo declarado por Sugden y Bairner (1991, pp. 133-141), “El deporte tiene un fuerte pedigrí político"; es decir, algunos deportes como el rugby y el hockey están asociados con la cultura británica, mientras que los deportes gaélicos representan la tradición irlandesa. Por tanto, es de vital importancia escoger con mucha sensibilidad las disciplinas que van a ser parte de las intervenciones sociales por medio del deporte y ser cuidadosos a la hora de introducir los deportes de cierta tradición a la comunidad contraria. Tal y como lo manifestó un funcionario de la ONG Football for Peace: “El deporte tiene que ser administrado cuidadosamente porque es parte del conflicto".

A diferencia de sus homólogos europeos, los funcionarios DDP colombianos reportaron una gama más amplia de fenómenos relacionados con el conflicto en Colombia que, creen, pueden abordarse utilizando en parte el deporte como un medio. En su opinión, el deporte puede ser útil para: 1) Proporcionar un espacio seguro para niñas, niños y jóvenes en estado de riesgo; 2) transmitir habilidades y destrezas que les permitan tomar decisiones adecuadas si los jóvenes se ven en riesgo de ser reclutados por fuerzas armadas ilegales o redes criminales; 3 ) ayudar a los beneficiarios a construir un proyecto de vida; 4) adquirir habilidades que les ayuden a generar empleos y oportunidades económicas; 5) fomentar la convivencia pacífica y la lucha contra la violencia urbana y 6) permitir la inclusión social.

Una coordinadora del programa Gestores del Deporte expresó su opinión sobre el uso del deporte para prevenir el reclutamiento ilegal de jóvenes:

Hay niñas, niños y jóvenes que han estado practicando deportes con nosotros desde hace algún tiempo; nosotros creemos que si en un caso les piden que se unan a un grupo armado ilegal, ellos están en condiciones de decir no. A menos, por supuesto, que se les amenace o algo por el estilo, pero si pueden elegir, ellos ahora tienen las herramientas para decir no.

El hecho de que los funcionarios hayan destacado una amplia lista de elementos que consideran específicos del conflicto colombiano y que podrían ser parcialmente abordados a través del deporte, revela algunos de los factores que, en su opinión, también son necesarios para lograr la paz. En este sentido, alcanzar la paz no consiste solamente en poner fin a las hostilidades armadas y promover una convivencia armoniosa entre los colombianos, sino que también en su opinión, se trata de crear oportunidades económicas y asistir a aquellos al margen de la sociedad para que, por sí mismos, desarrollen un proyecto de vida.

3) Con respecto al papel de los funcionarios DDP como promotores de paz y facilitadores de cambio comunitario, la encuesta en línea demostró que la mayoría de los funcionarios se perciben a sí mismos como constructores de paz. A la pregunta Considerando el trabajo que usted hace y teniendo en cuen- 
ta los objetivos de su organización, ¿Cree que ha hecho una contribución a la creación de una cultura de paz?, respuestas positivas en este frente en Irlanda del Norte incluyen la siguiente:

Sí. Estoy muy orgulloso de lo que he logrado hasta la fecha. El fútbol es la herramienta que hemos utilizado, pero también he complementado este trabajo con mis habilidades personales... (Entrenador y coordinador, Asociación Norirlandesa de Fútbol - IFA).

Las ramificaciones de respuestas como estas son múltiples, ya que, a través de estas reflexiones los funcionarios sugieren que: 1) Tienen una comprensión del conflicto y que sus esfuerzos y acciones están dirigidas a facilitar la paz; 2) son conscientes de las conexiones entre su trabajo, el papel del deporte y el contexto del conflicto en sus sociedades; 3) son conscientes de, y evitan, el posible impacto negativo de sus intervenciones y 4) están equipados con metodologías o herramientas específicas para maximizar su papel como constructores de paz.

Aunque repasar a fondo cada uno de los supuestos anteriormente señalados está fuera del alcance de este documento, vale la pena considerar la idea de que si los funcionarios DDP se ven a sí mismos como constructores de paz, estos, por ende, debieron haber adquirido ciertas habilidades que les permita cumplir a cabalidad esta tarea. Aunque fue evidente durante mis interacciones con los funcionarios DDP (especialmente en Colombia) que algunos de estos profesionales han desarrollado competencias útiles (al estar en estrecho contacto con comunidades marginadas, aprendiendo de las necesidades de las comunidades y adaptando el deporte para hacer frente a algunas de esas necesidades), la adquisición de habilidades adicionales a través de medios más estructurados sin duda puede agregar valor a su "trabajo por la paz". Al respecto, se puede anticipar la importancia de las instituciones académicas y los profesionales en el campo de la construcción de la paz y resolución de conflictos, ya que estos pueden complementar el conocimiento empírico adquirido por los funcionarios DDP, arrojando luz sobre la aplicación práctica de enfoques teóricos de construcción de paz y ayudando a los oficiales a localizar sus propios esfuerzos dentro del amplio campo de la construcción de paz y la resolución de los conflictos. Mientras que la cooperación entre la academia, los funcionarios y las ONG en el área de la paz y el desarrollo a través del deporte es bastante común en Irlanda del Norte (Universidad de Ulster, Universidad de Brighton), en Colombia tal colaboración aún está por suceder y se recomienda fuertemente.

\section{Dimensión II: Programas de DDP}

La segunda dimensión: 1) exploró los componentes deportivos y no deportivos de los programas del DDP; 2) reportó sobre las redes de cooperación entre oficiales de DDP en Colombia, señalando la forma en que algunas iniciativas se involucran con la comunidad vía programas de extensión en Irlanda del Norte y 3) proporcionó una visión general de cómo algunos de los programas del DDP se ubican dentro de objetivos más amplios de paz y desarrollo a nivel nacional y su repercusión en la formulación de política pública.

En términos generales, los programas de deporte para la paz y el desarrollo se dividen en dos categorías: Sport plus y Plus sport (Coalter, 2008). Los programas de Sport plus enfatizan la participación del público en actividades deportivas, lo que a su vez puede conllevar a la consecución de otros objetivos sociales (educación, salud, aumento de la participación ciudadana, entre otros). En los programas de Plus sport, el deporte es el vehículo primario para que las poblaciones en 
riesgo puedan alcanzar una serie de metas sociales más amplias como la paz y el desarrollo (Coalter, 2007). En ambos métodos, los resultados deseados no se alcanzan por medio del deporte por sí solo, sino que incorporan una variedad de prácticas no deportivas, tales como los juegos cooperativos, seminarios, talleres y otras actividades pedagógicas (Cárdenas, 2013).

1) Tanto en Colombia como en Irlanda del Norte, los programas del DDP se estructuran en torno a actividades deportivas y no deportivas. Las actividades no deportivas ayudan a consolidar las lecciones aprendidas a través del deporte, mientras que los mensajes positivos y las habilidades transmitidas a través de esta vía pueden aplicarse más allá de los campos deportivos. En Irlanda del Norte, actividades populares no deportivas incluyen seminarios sobre relaciones intercomunitarias y talleres de resolución de conflictos (Peace Players International); talleres de arte (Seaview Enterprises); discusiones guiadas sobre temas referentes al conflicto y el sectarismo (Teenage Kikz) y la identidad cultural (Universidad de Ulster).

En Colombia, el componente no deportivo de los programas de DDP incluye seminarios y talleres de arte, fotografía, danza y música, programas de promoción de jóvenes y excursiones (Red Fútbol y Paz) ${ }^{4}$; charlas sobre prevención del abuso de sustancias ilícitas, desarrollo comunitario, paz y seguridad (Gestores del Deporte); enseñanza de habilidades para la resolución de conflictos (Tiempo de Juego, Colombianitos); seminarios públicos sobre educación y civismo (Fundación Luker), entre otras actividades.

\footnotetext{
4 La Red Fútbol y Paz reúne a tres socios internacionales (La Agencia Alemana para la Cooperación Internacional - GIZ, el Programa de las Naciones Unidas para el Desarrollo en Colombia - PNUD, y el Banco Mundial) y a doce organizaciones no gubernamentales del DDP, seis de las cuales participaron en este estudio.
}

Además de lo anterior, el programa "A Ganar" enseña a sus beneficiarios una serie de habilidades necesarias para obtener trabajo en una variedad de campos, empleando una serie de actividades no deportivas como son 250 horas de formación profesional, una pasantía y servicio a la comunidad.

2) En cuanto a la forma en que los programas y funcionarios del DDP se involucran con sus respectivas comunidades y con otras fuera de sus áreas inmediatas de acción, se concluyó que las ONG y los funcionarios en el área del DDP están en capacidad de impulsar un cambio positivo a través de la formación de redes y el establecimiento de vínculos entre las comunidades y los actores políticos relevantes. En Colombia, la Red Fútbol y Paz ha sido pionera en el desarrollo de redes de cooperación entre funcionarios y organizaciones del DDP. Trabajando bajo un marco común y con el apoyo logístico y técnico del Programa de las Naciones Unidas para el Desarrollo en Colombia - PNUD, La Agencia Alemana para la Cooperación Internacional - GIZ y el Banco Mundial, doce programas y fundaciones se han unido con el fin de crear conciencia sobre el uso del fútbol como herramienta de desarrollo y paz, así como para abordar colectivamente -a través del deporte- problemáticas sociales que afectan a los niños y jóvenes en las comunidades donde los programas operan. A diferencia de Colombia, la creación de redes estratégicas entre funcionarios DDP no fue un tema predominante en el caso de Irlanda del Norte.

En Irlanda del Norte, las ONG más experimentadas han fortalecido lazos con la comunidad a través de programas de extensión y mediante el diseño y la instrucción de cursos universitarios abiertos a la comunidad en general. Peace Players International desarrolló un curso 
que incorpora un plan de estudios sobre las relaciones comunitarias en Irlanda del Norte, mientras que la Universidad de Ulster imparte el curso "Deporte para la Inclusión, la Actividad Física y la Recreación", el cual instruye a participantes en áreas tales como la construcción de la paz y la inclusión social por medio del deporte.

3) En materia de política pública, este estudio encontró que existe suficiente conciencia en ambas regiones sobre el importante papel que el deporte y que las intervenciones sociales basadas en el deporte pueden desempeñar dentro de los planes nacionales y regionales de desarrollo y paz. En Colombia, algunos programas del DDP implementados por el Estado (por ejemplo, "Gestores del Deporte" y "Golombiao") se han incrustado desde su creación dentro de los planes de desarrollo estatal y nacional. No obstante, solo hasta hace poco -cuando el Plan Decenal de Seguridad, Comodidad y Convivencia en el Fútbol 2014-2024 salió a la luzse reconocieron las contribuciones que las fundaciones, los programas y las metodologías del DDP han hecho a la construcción de paz en el país. Este constituye un importante avance para el posterior desarrollo de política pública concreta en torno a la función social del deporte en la nación.

En Irlanda del Norte -según lo demostrado en documentos clave como Sport Matters: The Northern Ireland Strategy for Sport \& Physical Recreation 2009-2019 y Programme for Cohesion, Sharing and Integration-, existe un claro entendimiento sobre la función del deporte como catalizador social, pero, también, como posible ventana para la expresión de sentimientos sectarios. El reconocimiento de estos dos aspectos del deporte demuestra que también hay una amplia conciencia de la condición política del deporte en esta región. No obstante, hay aún una clara ausencia de política pública en torno al papel del deporte dentro del contexto del conflicto en Irlanda del Norte. Al respecto, un funcionario de la Universidad de Ulster comentó:

Decisiones importantes podrían hacerse con base en estos dos documentos. EI tema del deporte y las relaciones entre católicos y protestantes se menciona en ellos, pero ninguno toma el liderazgo en esta área. Esta es una de las áreas donde el gobierno tiene que tomar posesión y liderar procesos.

\section{Dimensión III: El sector del DDP}

La tercera dimensión examinó el sector del deporte para la paz y el desarrollo en Colombia e Irlanda del Norte y proporcionó algunas ideas preliminares sobre el nivel de cohesión e integración entre los principales actores en el mundo del DDP. Darnell (2012) sostiene que hay literalmente cientos de organizaciones que forman el sector del DDP, quienes movilizan el deporte de un modo u otro para lograr objetivos explícitos de desarrollo. Las naciones, las ONG, las organizaciones intergubernamentales, las federaciones deportivas internacionales, las empresas transnacionales y las organizaciones comunitarias son instituciones clave dentro del sector del DDP (Giulianotti, 2011a, p. 208).

Algunas conclusiones preliminares fueron extraídas de la comparación y el contraste en el sector del DDP en ambas regiones. El sector del DDP en Irlanda del Norte exhibe un nivel satisfactorio de integración con múltiples oportunidades -tanto físicas como virtuales- para que los actores principales en esta área y el público en general interactúen, promoviendo el intercambio de información en este campo dentro y fuera de este territorio. Dada la fuerte influencia de instituciones e individuos anglosajones en el campo del DDP global, los oficiales y las organizaciones en Irlanda del Norte tienen acceso 
a una comunidad más amplia de actores que promueven el DDP (por ejemplo, por medio de programas académicos, foros, conferencias y plataformas virtuales como Sportanddev $)^{5}$. Por el contrario, en Colombia -al igual que en el resto de América Latina- el DDP todavía no es un campo consolidado.

\section{Reflexiones finales y recomendaciones}

Las reflexiones de los funcionarios del DDP en Colombia e Irlanda del Norte indican que las intervenciones y programas para el fomento de la paz por medio del deporte han hecho una contribución modesta, pero tangible, a la construcción de la paz en estas sociedades afectadas por la división y el conflicto. En Irlanda del Norte, programas e intervenciones basados en el deporte recreativo han proporcionado una plataforma para promover el contacto entre niños, jóvenes y adultos de comunidades en conflicto, con la esperanza de que relaciones significativas pueden ser creadas y mantenidas a través de la brecha que divide a protestantes y católicos. En Colombia, el deporte ha contribuido a los esfuerzos de consolidación de la paz, creando un espacio donde aquellos que se encuentran a las márgenes de la sociedad puedan jugar, recrearse y aprender una serie de habilidades útiles y donde pueden, en cierta medida, desarrollar un plan de vida para ellos mismo lejos de la ilegalidad. Este estudio también ha demostrado que el contexto donde tienen lugar las intervenciones y la dinámica del conflicto juega un papel crucial en la formación de las percepciones de los funcionarios del DDP con respecto a su propio trabajo y la capacidad y las limitaciones del deporte como vehículo para la paz.

Basados en la discusión que se ofrece en este documento, hay una serie de

5 Sportanddev es un repositorio de información y plataforma virtual sobre el DDP: http://www.sportanddev.org/ recomendaciones en relación con el deporte y la construcción de la paz dirigida a funcionarios del DDP y organizaciones en Colombia e Irlanda del Norte. Estas recomendaciones incluyen:

1) Tras el caso de Irlanda del Norte, se recomienda que los funcionarios del DDP en sociedades divididas estén al tanto de elementos culturales o políticos subyacentes asociados con el deporte que puedan disminuir su eficacia -o incluso, exacerbar las animosidades entre grupos en conflicto- cuando este se utiliza como una herramienta de cohesión social y paz.

2) Las actividades no deportivas juegan un papel importante en los programas de DDP y, por lo tanto, estas deben ser cuidadosamente seleccionadas e incorporadas dentro de las intervenciones deportivas; un equilibrio entre las actividades deportivas y no deportivas debe guiar los programas de deporte para la Paz. Del mismo modo, el deporte por sí mismo no debe ser considerado como una estrategia integral para el fomento de la paz. Las intervenciones del DDP pueden trabajar más eficazmente con el apoyo de otras estrategias e idealmente, deberían operar dentro de los planes de construcción de paz más amplios.

3) Es altamente recomendada la colaboración entre instituciones académicas, los profesionales del sector de la paz y la resolución de conflictos y las organizaciones del DDP; esto, con el fin de ayudar a los funcionarios de programas del DDP a incrementar sus habilidades como "constructores de paz".

4) La formación de redes estratégicas entre los funcionarios DDP puede ayudar significativamente a impulsar este sector. Para que esto suceda, trabajar bajo un marco común y desarrollar 
una visión compartida es esencial. En el mejor de los escenarios, estas redes pueden conllevar a la creación de una mayor conciencia entre las agencias gubernamentales sobre la importancia de apoyar las intervenciones sociales basadas en el deporte.

5) Teniendo en cuenta la difícil tarea de crear política pública en una sociedad dividida como lo es Irlanda del Norte, se recomienda que las instituciones encargadas de esta materia establezcan políticas claras que guíen al sector del deporte para la paz y el desarrollo en este territorio.

6) Para que el DDP opere como un campo auténtico en Colombia, es necesario que existan vías -tanto físicas como virtuales- para difundir información, compartir las mejores prácticas y experiencias, desarrollar programas académicos, crear una comunidad de participantes comprometidos con la promoción del deporte como catalizador social y aumentar el nivel de integración entre los actores que promueven el DDP.

7) Conforme Colombia entra lentamente en su era posconflicto, es recomendable que los programas y las intervenciones sociales basados en el deporte sean rediseñados para hacer frente a los retos que esta fase implica. Es de particular interés explorar las formas en que el deporte pueda asistir a la reintegración de jóvenes combatientes a la vida civil y a la creación de oportunidades económicas y de rehabilitación psicológica y física a las víctimas de la guerra.

\section{Referencias}

Allport, G. W. (1954). The nature of prejudice. Cambridge, MA: Addison-Wesley.

Cairns, E., \& Hewstone, M. (2002). Northern Ireland: The impact of peacemaking in Northern Ireland on intergroup behavior. In G. Salomon \& B. Neov (Eds.). Peace education: The concept, principles and practices around the world (pp. 217-228). Hilldale, NJ: Lawrence Erlbaum Associates.
Cárdenas, A. (2012). El proyecto Goles por la paz en Colombia y las Filipinas: un acercamiento al uso de los deportes y los juegos cooperativos para la paz. Revista de Educación Física para la Paz, 7(1), 12-23.

Cárdenas, A. (2013). Peace building through sport? An introduction to sport for development and peace. Journal of Conflictology, 4(1), 24-33.

Cárdenas, A. (2015a). Sport, the international community and the peace talks in Colombia. Recuperado de http://watch.peace-sport.org/opinion/sport-theinternational-community-and-the-peace-talks-incolombia/

Cárdenas, A. (2015b). Sport for peace in a post-conflict Colombia. Recuperado de http://www.blogs.hss. ed.ac.uk/sport-matters/2015/08/13/sport-for-peacein-a-post-conflict-colombia/

Coalter, F. (2007). A wider social role of sport: Who's keeping the score? Abingdon, England: Routledge.

Coalter, F. (2008). Sport-in-development: A monitoring and evaluation manual. Stirling, UK: University of Stirling. Recuperado de http://www.iscaweb. org/files/Active_Network_2013/Desk_Research/9 sport_in_devēlopment_a_monitoring_and_ evaluation manual.pdf

Creswell, J. W. (1998). Qualitative inquiry and research design: Choosing among five traditions. Thousand Oaks, CA: SAGE.

Creswell, J. W. (2003). Research design: Qualitative, quantitative, and mixed methods approaches $(2 \mathrm{a}$ ed.). Thousand Oaks, CA: SAGE.

Darnell, S. (2011). Sport for development and peace: $A$ critical sociology. London, England: A\&C Black.

Department of Culture, Arts and Leisure (DCAL) - Sport NI. (2009). Sport matters: The Northern Ireland strategy for sport \& physical recreation 2009-2019. Belfast, Northern Ireland: Department of Culture, Arts and Leisure - House of Sport. Recuperado de http://www.dcalni.gov.uk/sport matters.pdf

Giulianotti, R. (2011a). Sport, transnational peacemaking, and global civil society: Exploring the reflective discourses of "sport, development, and peace" project officials. Journal of Sport and Social Issues, 35(1), 50-71.

Giulianotti, R. (2011b). The sport, development and peace sector: A model of four social policy domains. Journal of Social Policy, 40(04), 757-776.

Giulianotti, R., \& Armstrong, G. (2011). Sport, the Military and peacemaking: History and possibilities. Third World Quarterly, 32(3), 379-394.

Hillyer, S. J. (2013). Sport \& peace: Mapping the field. Washington, DC: Georgetown University. Recuperado de http://idrottsforum.org/wpcontent/ uploads/2013/11/Sport-Peace-Mapping-the-Field-2. pdf

Instituto Colombiano del Deporte - COLDEPORTES. (2009). Plan decenal del deporte, la recreación, la educación física y la actividad física, para el desarrollo humano, la convivencia y la paz 20092019. Bogotá, Colombia: Editorial ABC. Recuperado de https://fundamentosadministracion.files. wordpress.com/2012/08/plan-decenalcoldeportes. pdf

Kay, T., \& Dudfield, O. (2013). The commonwealth guide to advancing development through sport. London, UK: Commonwealth Secretariat.

Kidd, B. (2008). A new social movement: Sport for development and peace. Sport in Society, 11(4), 370-380.

McGinley, M., Kremera, J., Trewa, K., \& Ogle, S. (1998). Socio-cultural identity and attitudes to sport in Northern Ireland. The Irish Journal of Psychology, 19(4), 464-471.

McGlynn, C. (2010). Culture and peace-building in integrated schools in Northern Ireland (Fostering Peace through Cultural Initiatives from the 
Roundtable on Conflict and Culture). Tokyo, Japan: Joint Research Institute for International Peace and Culture (JRIPEC), Aoyama Gakuin University, The Japan Foundation London. Recuperado de http:// www.jripec.aoyama.ac.jp/english/publication/pdf/ fostering_peace_through_cultural_initiatives03.pdf

Merriam, S. B. (1998). Qualitative research and case study applications in education. San Francisco, CA: Jossey-Bass.

Schulenkorf, N. (2010). Sport events and ethnic reconciliation: Attempting to create social change between Sinhalese, Tamil and Muslim sportspeople in war-torn Sri Lanka. International Review for the Sociology of Sport, 45(3), 273-294.

Sport for Development and Peace International Working Group - sdp iwg. (2008). Harnessing the power of sport for development and peace: recommendations to governments. Toronto, Canada: Right to Play. Recuperado de http://www. un.org/wcm/webdav/site/sport/shared/sport/pdfs/ SDP\%20IWG/Final\%20SDP\%20IWG\%20Report. pdf

Stake, R. (2000). Case Studies. In N. K. Denzin \& Y. S. Lincoln (Eds.). Handbook of qualitative research (2a ed.) (pp. 435-454). Thousand Oaks, CA: SAGE.

Sugden, J., \& Bairner, E. S. (1991). The Political Culture of Sport in Northern Ireland. Studies: An Irish Quarterly Review, 80(318), 133-141.

Yin, R. (Ed.). (2004). The case study anthology. Thousand Oaks, CA: SAGE.

Yin, R. (2009). Case study research: Design and methods (4a ed.). Thousand Oaks, CA: SAGE. 